\title{
Effect of Specimen Shape on Fatigue Behavior in Thin Pure Copper Sheet for Smart Stress-memory Patch
}

\author{
Takayuki SHIRAIWA* and Manabu ENOKI \\ Department of Materials Engineering, The University of Tokyo, Tokyo, 113-8656 Japan. \\ (Received on April 28, 2014; accepted on June 2, 2014)
}

\begin{abstract}
Smart stress-memory patch is a promising method for monitoring long-term fatigue damage in structure. The patch can estimate the number of cycles and the stress amplitude using fatigue crack growth properties of thin metal sheets. In this study, fatigue crack growth behavior of thin pure copper sheet was investigated by changing the specimen shape under strain-controlled testing in order to improve measuring range of smart stress-memory patch. To characterize the initiation as well as the stable growth of fatigue cracks, the relationship between stress intensity factor range and crack growth rate was successfully fitted to one equation regardless of strain amplitude, strain ratio and specimen shape. Based on the experimental results, an equation for estimating fatigue cycles from fatigue crack length was derived. In addition, the detectable range of stress amplitude was evaluated and its dependence on sensor shape and stress ratio was shown. Since this patch needs neither power supply nor wiring, it provides a great potential for long-term structural health monitoring with easy maintenance.
\end{abstract}

KEY WORDS: structural health monitoring; fatigue sensor; fatigue crack growth; Paris law; electrodeposited copper.

\section{Introduction}

In recent years, aging and deterioration of infrastructure pose a significant risk in Japan and other developed countries, and the situation becomes critical due to shortage of experienced inspector and rapid increase of aged infrastructures. Under these circumstances, structural health monitoring (SHM) has received great attraction because of its great potential to monitor and maintain the safety of civil infrastructures such as bridges, ships, trains, aircraft, power plants and industrial machines. ${ }^{1-3)}$ The technologies related to SHM are classified into three categories: (1) sensing, (2) data acquisition, and (3) information processing. Over the past decade, there have been large amounts of research in the area of wireless networking and computing toward (2) data acquisition and (3) information processing, which facilitate the implementation of SHM in infrastructures. ${ }^{4,5)}$ However, the sensing technology in wireless SHM has made relatively small advances, and most of the latest SHM systems still utilize traditional accelerometer and strain gauge. These sensing methods have a number of problems on the practical use such as necessity of wiring, electrical power supply and complicated measuring devices. Especially when evaluating the fatigue damage, they require to monitor the stress continuously with a high-speed communication throughout the in-service life and to analyze the huge volumes of data.

Concept of smart stress-memory patch (hereinafter called

\footnotetext{
* Corresponding author: E-mail: shiraiwa@rme.mm.t.u-tokyo.ac.jp
} DOI: http://dx.doi.org/10.2355/isijinternational.54.2342 "smart patch") was proposed to overcome above problems in the previous papers. ${ }^{6-11)}$ Sensor in smart patch consists of a thin metal sheet with a pre-crack, and number of fatigue cycles and stress amplitude on structure can be estimated from fatigue crack growth in the sensor. Since the accumulation of the fatigue damage is memorized in the sensor itself, it requires no power supply, wiring, continuous monitoring or high-speed communication. Furthermore, the patch is successfully applied to batteryless wireless system to measure the crack length in the sensor using radio frequency identification (RFID) technique. It has been demonstrated that stress amplitude and cyclic number of a steel bar can be estimated from the crack length of the sensor using equations based on Paris law. However, the measurement range of stress amplitude is limited from $100 \mathrm{MPa}$ to $200 \mathrm{MPa}$ when the smart patch is attached to steel materials. ${ }^{8)}$ To apply various stress conditions, the measurement range of stress should be further extended.

In order to modify the sensor characteristics of the smart patch, the fatigue crack growth behavior in the thin metal sheet should be investigated. While most studies about fatigue in thin metal have focused on stress-life (S-N) and strain-life $(\varepsilon-\mathrm{N})$ curves for reliability of microelectronic products, ${ }^{12-14)}$ the fatigue crack growth behavior under strain-controlled testing has not been enough explored in detail. In our past research, it has been shown that electrodeposited copper (ED-Cu) with fine-grained microstructure $(\sim 2 \mu \mathrm{m})$ offers stable crack propagations and the scattering in the crack growth rate was enough small to estimate cyclic numbers. ${ }^{6}$ However, the effect of the sensor shape on the crack growth behavior has not been revealed. 
In this study, fatigue crack growth behavior of the sensor (thin pure copper sheet) under uniaxial strain-controlled fatigue testing was evaluated changing the sensor shape and strain ratio. The scattering in crack growth was also examined by a stochastic model to assess the error in estimation of fatigue cycles and stress amplitude. From these observations, the measurement range of stress amplitude in the smart patch was investigated as the function of the sensor shape and strain ratio.

\section{Principle of Smart Stress-memory Patch}

Figure 1 provides an illustrative description of the principle of the smart patch. The sensor in the patch consists of a thin metal sheet with a pre-crack. Two or more sensors are attached on structure, for example, close to the welded part (Fig. 1(a)). In most cases, the function of fatigue crack growth in the sensor is described by the following equation:

$$
\frac{d a}{d N}=f(\Delta K, R),
$$

where $d a / d N$ is crack growth rate, $\Delta K$ is stress intensity factor range, $R$ is stress ratio. Then, the number of fatigue cycles $(N)$ and stress amplitude $(\Delta \sigma)$ on the structure can be estimated from fatigue crack growth curves of two sensors with different characteristics, as shown in Fig. 1(b). The details of the derivation of stress amplitude and cyclic number are given in the previous paper. ${ }^{10)}$

\section{Experimental Procedure}

\subsection{Materials}

The sensors were made of electrodeposited (ED) copper produced by Furukawa Electric Co., Ltd., since electrodeposited materials tend to have a very small grain size and provide stable crack propagations. ${ }^{6,15)}$ Microstructural observations revealed that the ED-Cu has a relatively equiaxed grain structure with an average grain size of approximately $2 \mu \mathrm{m}$.

\subsection{Specimen (Sensor in Smart Patch)}

Four different geometries were considered as a sensor shape in smart patch, as shown in Fig. 2. The ED-Cu sheet with a thickness of $0.1 \mathrm{~mm}$ was cut to rectangular coupons with a dimension of $40 \mathrm{~mm} \times 5 \mathrm{~mm}, 40 \mathrm{~mm} \times 10 \mathrm{~mm}$ and $60 \mathrm{~mm} \times 10 \mathrm{~mm}$. Side groove was fabricated by chemical etching with a solution mainly composed of ferric chloride

(a)

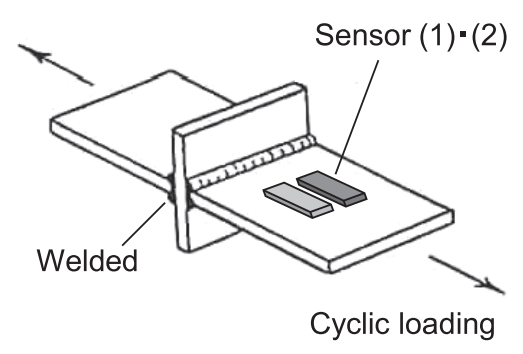

(H-1000A, Sunhayato Corp.). The etching time was fixed at 10 minutes, and the thickness between top and bottom of groove $\left(T_{\mathrm{c}}\right.$ shown in Fig. 2(d)) was varied within 65 to $75 \mu \mathrm{m}$. A single notch with a length of $2.5 \mathrm{~mm}$ and a width of $0.3 \mathrm{~mm}$ was induced at the center from one side of the coupon. The notch tip was round-shaped with a radius of approximately $150 \mu \mathrm{m}$. Additionally, the notch tip was sharpened to curvature radius of about $30 \mu \mathrm{m}$ by a razor blade (High-stainless $100 \mu \mathrm{m}$, Feather Safety Razor Co., Ltd.). One surface of the coupon was polished to a mirror finish using $3 \mu \mathrm{m}$ and $1 \mu \mathrm{m}$ alumina slurry to observe crack length clearly. Afterward, fatigue pre-crack was introduced under the conditions of a maximum cyclic stress of $24 \mathrm{MPa}$ and a stress ratio of 0.1 until the crack length reached $2.7 \mathrm{~mm}$ from the edge.

\subsection{Fatigue Test}

Fatigue tests were performed at room temperature using
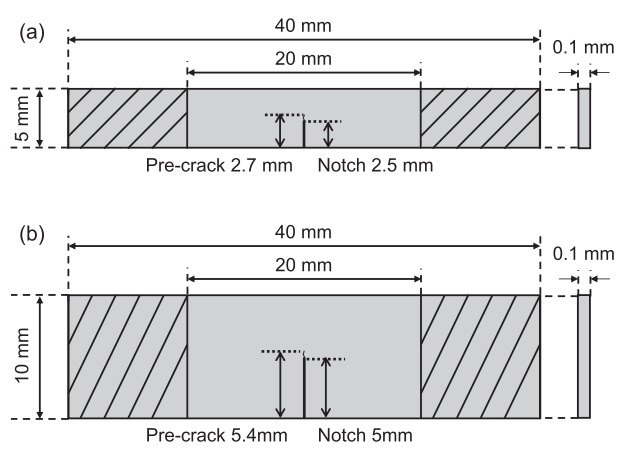

(c)

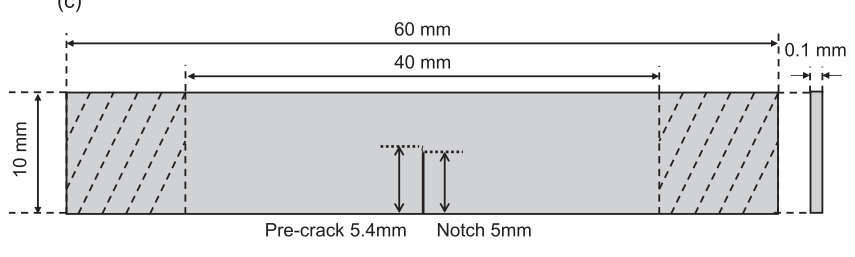

(d)

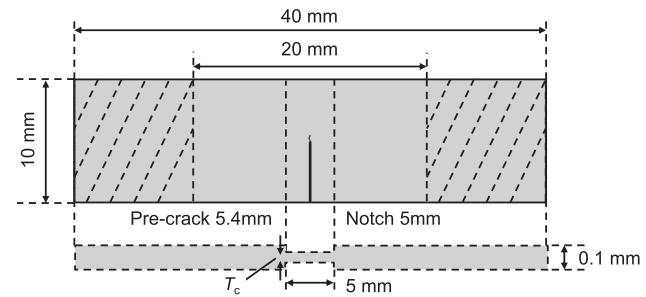

Fig. 2. Shape of (a) smooth specimen (W5-H20); (b) smooth specimen (W10-H20); (c) smooth specimen (W10-H40); (d) $30 \%$-side-grooved specimen (W10-H20). (b)

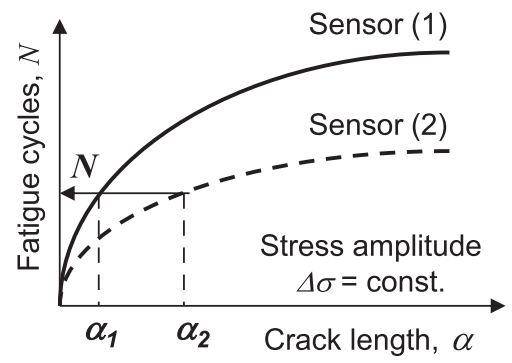

Fig. 1. Principle of smart stress-memory patch: (a) installation on the structure; (b) estimation of cyclic number from crack length of the sensor. 
electromagnetic fatigue testing machines (MMT-100N and MMT-500N, Shimadzu). Both sides of the sensor were clamped to the machine grips, and the distance between the upper and lower jigs was adjusted to match the gage length shown in Fig. 2. Then, the constant-amplitude cyclic loading was applied to all specimens under displacement control with a maximum strain of 0.05 to $0.3 \%$, frequency of $19 \mathrm{~Hz}$, and strain ratio of $R=0.5$. The strain was defined as a crosshead displacement divided by gauge length of specimen.

In addition, $\Delta K$-decreasing and $\Delta K$-increasing tests for smooth specimen with length of $5 \mathrm{~mm} \times 20 \mathrm{~mm}(\mathrm{~W} 5-\mathrm{H} 20)$ were carried out under strain ratio of $R=0.1$ and 0.3 according to ASTM E647 ${ }^{16)}$ in order to investigate the effect of strain ratio on the crack growth. During fatigue testing, the crack length in the sensor was measured optically at intervals of 30 seconds with a traveling microscope (VHX-600, Keyence).

\section{Analytical Procedure}

\subsection{Stress Intensity Factor}

It is commonly known that the stress intensity factor of single edge-cracked tension specimen with prescribed end displacements can be expressed by ${ }^{17)}$

$$
K=\sigma \sqrt{\pi a} \cdot g(\alpha, \beta), \sigma=\frac{E u}{L}, \alpha=\frac{a}{W}, \beta=\frac{L}{W},
$$

where $g(\alpha)$ is shape factor, $W$ is width of the specimen, $L$ is gauge length of the specimen, $a$ is crack length, $E$ is Young's modulus, $u$ is displacement on the specimen, $\alpha$ is normalized crack length and $\beta$ is ratio of the gauge length to the width. The shape factor for the present sensor geometry was calculated by finite element method (FEM) to examine the fatigue crack growth characteristics as a function of stress intensity factor range. The calculated results are shown in Fig. 3. These shape factors can be fitted as follows:

$$
\begin{aligned}
& g(\alpha)=0.938-2.62 \alpha+3.77 \alpha^{2}-1.90 \alpha^{3} \\
& \text { (Smooth specimen } \beta=0.5 \text { ), }
\end{aligned}
$$

$$
g(\alpha)=1.123-2.26 \alpha+2.53 \alpha^{2}-1.04 \alpha^{3}
$$

(Smooth specimen $\beta=1$ ),

$g(\alpha)=1.002-0.040 \alpha-1.52 \alpha^{2}+1.09 \alpha^{3}$

(Smooth specimen $\beta=2$ ),

$$
g(\alpha)=0.896-1.43 \alpha-2.71 \alpha^{2}+1.02 \alpha^{3}
$$

(Smooth specimen $\beta=4$ ),

$g(\alpha)=1.036+1.06 \alpha+1.87 \alpha^{2}-3.21 \alpha^{3}$

(Smooth specimen $\beta=8$ ),

$$
g(\alpha)=1.241-0.64 \alpha-0.70 \alpha^{2}-0.81 \alpha^{3}
$$

(30\%-side-grooved specimen $\beta=2$ ).

Shape factors of smooth specimens increased with increasing aspect ratio, $\beta$. Calculated results from boundary element method (BEM) in reference ${ }^{18)}$ are also given in the same figure for comparison, which are in good agreement with the finite element results.

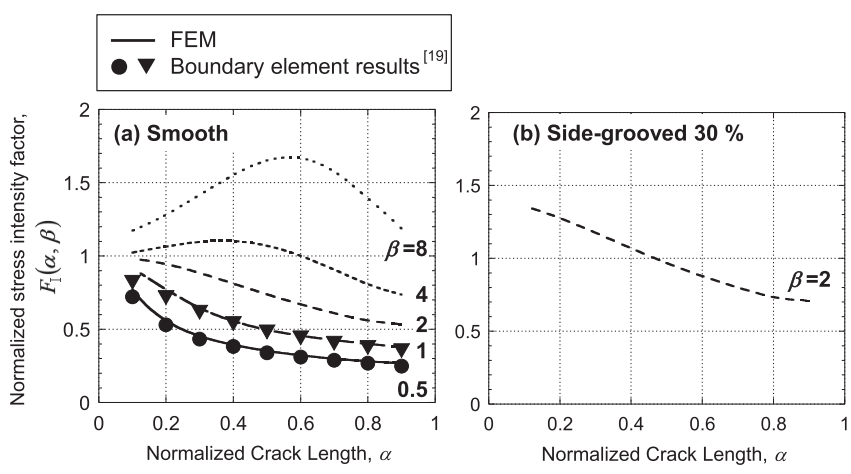

Fig. 3. Normalized stress intensity factor calculated by FEM. (a) Smooth specimen; (b) side-grooved specimen.

\subsection{Function Describing Fatigue Crack Growth Curve}

Although numerous functions for describing the fatigue crack growth have been proposed, three functions were considered in the current study. The Paris law ${ }^{19,20)}$ (Eq. (9)) and the Klesnil-Lukas relation ${ }^{21)}$ (Eq. (10)) are used to analyze the experimental data of constant-amplitude fatigue testing with $R=0.5$ :

$$
\begin{gathered}
\frac{d a}{d N}=C \Delta K^{m}, \ldots \ldots . \\
\frac{d a}{d N}=C\left(\Delta K^{m}-\Delta K_{t h}^{m}\right),
\end{gathered}
$$

where, $C, m$ are material constants and $\Delta K_{t h}$ is the threshold value of stress intensity factor range. These functions were used for analyzing the effect of specimen shape and strain amplitude on crack growth behavior in the near-threshold regime and Paris region. Then, an equation proposed by Kohout $^{22)}$ was employed to analyze the result of all data with $R=0.1$ to 0.5 :

$$
\frac{d a}{d N}=C\left[\left\{\frac{\Delta K}{(1-R)^{1-\beta_{w}}}\right\}^{m}-\Delta K_{t h 0}^{m}\right]
$$

where, $C, m, \beta_{w}$ are material constants and $\Delta K_{t h 0}$ is the threshold value of stress intensity factor range with $R=0$. This Kohout equation was utilized to access the effect of strain ratio on the crack growth and measurement range of the smart patch.

\subsection{Stochastic Analysis of Fatigue Crack Growth}

Among many stochastic models of the scattering in the fatigue crack growth, a simple model reported by Ortiz ${ }^{23,24}$ is used in this study, because it is valid in the case with various R-ratios, ${ }^{25)}$ and relatively simple to use in any function of fatigue crack growth. The model is described by the following equation:

$$
\frac{d a}{d N}=Z \cdot f(\Delta K, R),
$$

where $Z$ is random variable. If $\log Z$ is subjected to a normal distribution with zero mean, the variance of $\log Z$ represents the quantity of the scattering in the experimental fatigue crack growth rates. 

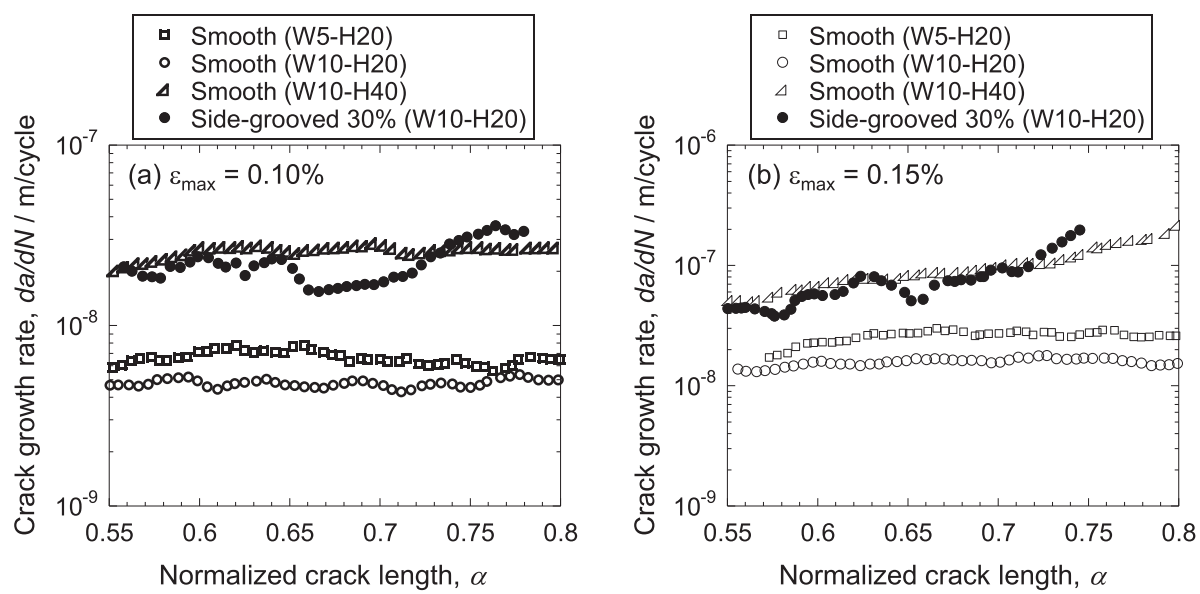

Fig. 4. Fatigue crack growth rate as a function of the normalized crack length for ED-Cu specimens with four shapes.

\section{Results}

\subsection{Fatigue Crack Growth under Strain Ratio of $\boldsymbol{R}=\mathbf{0 . 5}$}

Fatigue crack growth behavior in thin pure copper sheet was evaluated under constant-amplitude fatigue testing to study the effect of the sensor shape. Figure 4 shows fatigue crack growth rate under strain ratio of $R=0.5$ as a function of the normalized crack length. The crack growth rates were determined by the incremental polynomial method recommended in ASTM E 647. ${ }^{16)}$ The crack growth rate of smooth specimen (open symbols in Fig. 4) was almost constant with crack extension in the range of $\alpha=0.55$ to 0.8 , and that of side-grooved specimen (closed symbol) exhibited the same tendency as the smooth one, but fluctuated slightly throughout testing. The crack growth rate increased with the sensor length, and that of side-grooved specimen showed higher value than that of smooth specimen.

The relationship between stress intensity factor range calculated from FEM and experimental crack growth rate is shown in Fig. 5. The fitting curve was analyzed by the Paris Eq. (9) and the Klesnil-Lukas Eq. (10). In order to avoid underestimating the value of $\Delta K_{t h}$ in the Klesnil-Lukas equation, the inverse function to function (13) was used in fitting procedure:

$$
\Delta K=\left(\frac{d a / d N}{C}+\Delta K_{t h}^{m}\right)^{1 / m} .
$$

As shown in the figure, all data of four types of specimen belong to only one curve. The Paris equation (solid line in Fig. 5) is valid for intermediate $\Delta K$ levels ranging crack propagation rates from approximately $5 \times 10^{-9}$ to $10^{-7} \mathrm{~m} /$ cycle. On the other hand, the Klesnil-Lukas equation (dashed line) allows a successful fit of the greater part of the experimental crack growth curve including near the threshold value, and the fitted values were $C=5.72 \times 10^{-11}, m=2.87, \Delta K_{t h}=2.53$ $\mathrm{MPa} \cdot \mathrm{m}^{1 / 2}$. This threshold value of stress intensity factor range is related to the minimum value of detectable stress amplitude, as will be discussed later. Thus, it was shown that the crack growth behavior of the sensor is represented by a single curve regardless of strain amplitude and specimen shape.

\subsection{Fatigue Crack Growth under Strain Ratio of $\boldsymbol{R}=$ 0.1 to 0.5}

In order to clarify the dependence of the strain ratio, $\Delta K$ -

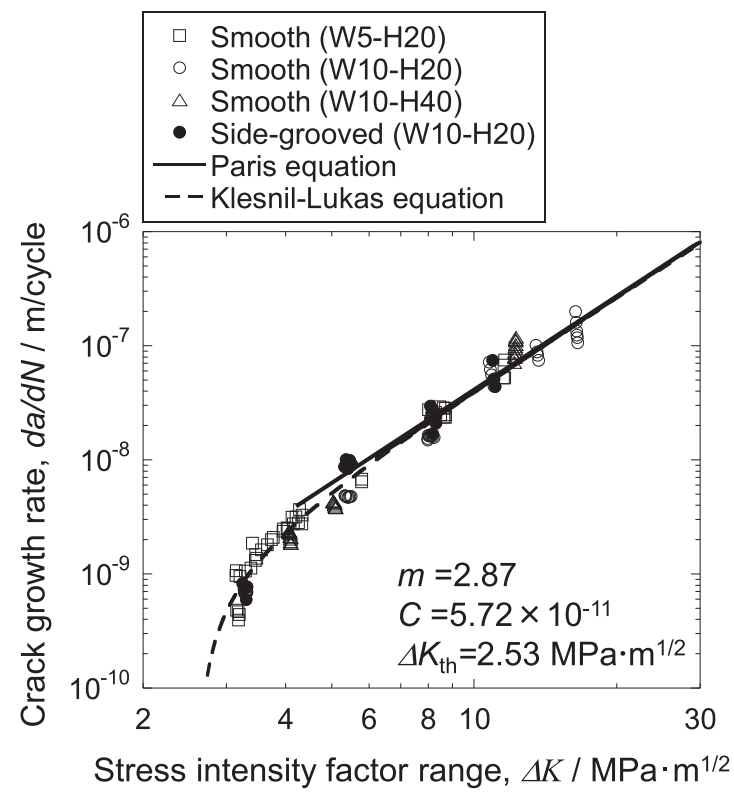

Fig. 5. Relationship between stress intensity factor range and crack growth rate for ED-Cu specimens with four shapes under strain ratio of 0.5 .

decreasing and $\Delta K$-increasing tests with smooth specimen (W5-H20) were conducted under strain ratio of $R=0.1$ and 0.3 . The results of fatigue crack growth under different strain ratio are plotted in Fig. 6. The results of constantamplitude testing with $R=0.5$ are also plotted in the same figure, and used in the fitting procedure. It can be seen that the crack growth of the sensor is substantially affected by the strain ratio, and the Kohout Eq. (11) provides a good fit. The fitted parameters are $C=1.16 \times 10^{-11}, m=2.87, \Delta K_{t h 0}=$ $4.41 \mathrm{MPa} \cdot \mathrm{m}^{1 / 2}, \beta_{w}=0.198$.

\subsection{Scattering on Fatigue Crack Growth}

The experimental results of constant-amplitude fatigue testing (Fig. 5) was used to analyze the scattering in fatigue crack growth. Substituting the Klesnil-Lukas function (10) to the Ortiz's stochastic model (12), $\log Z$ was obtained by each experimental data as follows:

$$
\log Z_{i}=\log \left(\frac{d a}{d N}\right)_{i}-\log C-\log \left(\Delta K_{i}^{m}-\Delta K_{t h}^{m}\right) .
$$

where $i$ is index number of experimental data and $n$ is the 


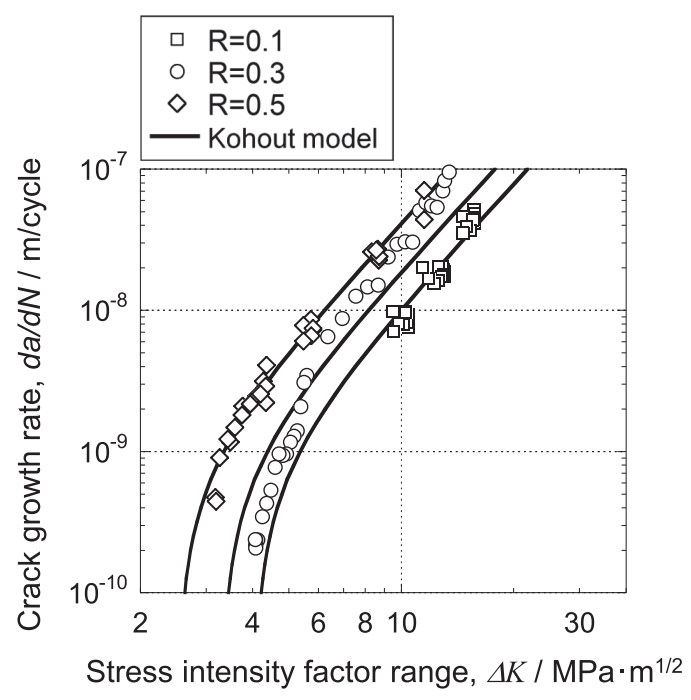

Fig. 6. Relationship between stress intensity factor range and crack growth rate for smooth specimen (W5-H20) with four shapes under strain ratio of 0.1 to 0.5 .

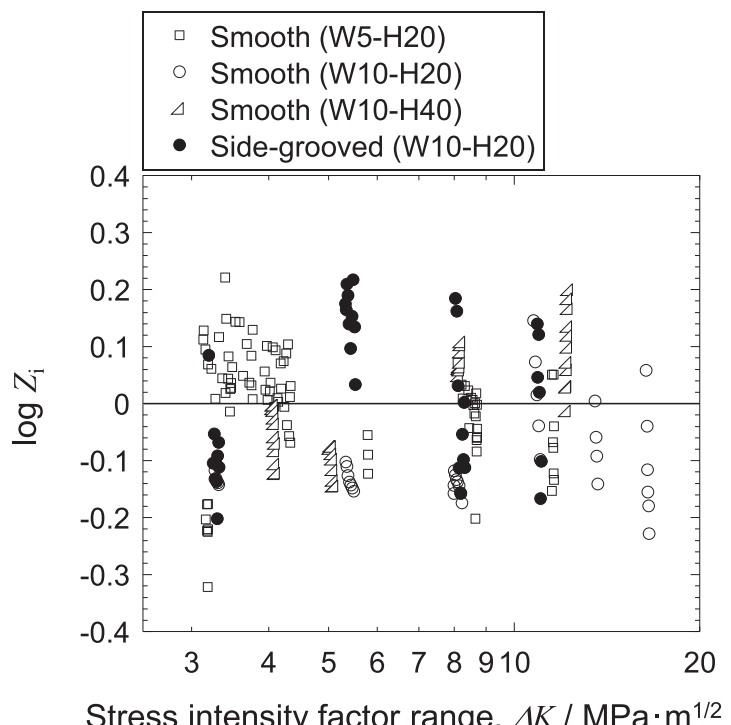

Fig. 7. $\log Z$ obtained from Eq. (13) as a function of stress intensity factor range.

total number, respectively. Figure 7 shows the $\log Z$ obtained from Eq. (14) as a function of stress intensity factor range, where there is no significant difference in scattering between the four specimen shapes. In this figure, the mean value of all data was almost zero and standard deviation $(\sigma \mathrm{SD})$ was 0.108 , which is enough smaller than that of rolled Cu specimen. ${ }^{6}$

\section{Discussion}

\subsection{Fatigue Crack Growth Behavior}

The fatigue crack growth rate of the sensor changed depending on the shape, as shown in Fig. 4. This is because the shape factor of the sensor increased with increasing aspect ratio $(\beta)$ and presence of side-groove (Fig. 3). Meanwhile, it is notable that all data of fatigue crack growth behavior in four types of specimen can be fitted to a single Eq. (10) regardless of its shape (length, width and groove), as shown in Fig. 5. This feature is a little different from several specimens reported in prior studies, ${ }^{26-28)}$ where side- groove promotes the retardation in fatigue crack growth in $\Delta K-d a / d N$ plot. The reason why the crack growth rate of side-grooved sensor in the smart patch is identical with that of the smooth sensors can be explained in terms of specimen thickness. The sensor in the smart patch is quite thinner than standard specimens and the region near the crack front is assumed to be under plane stress condition. For this reason, the effect of plastic constraint around the groove is less than the samples used in the references (for example, CT specimens used in reference ${ }^{26)}$ ). These results indicate that the crack growth rate of thin specimen that has the other shape parameters such as length, width and groove can be predicted based on shape factor calculated by FEM and material constants. It allows us to easily control the sensitivity of stress measurement in the smart patch by changing the sensor shape.

The side-grooved specimen (closed symbol in Fig. 4) exhibited fluctuation of fatigue crack growth rate throughout testing. A possible explanation of this fluctuation is the presence of surface imperfections due to the etching process such as thickness variations and surface roughness. The scattering analysis showed that the effect of this fluctuation is enough small.

The effect of strain ratio on the crack growth was also considered using the additional parameter, $\beta_{w}$, in the fatigue crack growth function. Crack growth behavior of smooth specimen (W5-H20) under strain ratio of $R=0.1,0.3$ and 0.5 was well fitted to this equation, as shown in Fig. 6 . Therefore, relationship between strain ratio and measurement range of the smart patch could be expressed by this equation.

\subsection{Prediction of Fatigue Cycles}

Based on fatigue testing results, a method to predict the fatigue cycles in structure using smart patch will be discussed. When the patch is attached to structure, the sensor is subjected to strain-controlled loading under the change in strain of the structure. It is assumed that the far-field stress is a uniaxial tension $\left(\sigma_{\text {structure }}\right)$ in elastic region and the sensor is perfectly bonded on the structure. If thickness of the sensor is enough thin and strain distribution in the structure is uniform regardless of the presence of the sensor, strain amplitude of sensor $\left(\Delta \varepsilon_{\text {sensor }}\right)$ and cyclic number of sensor $\left(N_{\text {sensor }}\right)$ are described as follows:

$$
\begin{gathered}
\Delta \varepsilon_{\text {sensor }}=\frac{\Delta \sigma_{\text {structure }}}{E_{\text {strucutre }}}, \\
N_{\text {sensor }}=N_{\text {structure }}, . .
\end{gathered}
$$

where $E_{\text {strucuture }}$ is Young's modulus of structure, $N_{\text {strucuture }}$ is cyclic number of structure, respectively. Using Kohout equation (Eq. (11)), the fatigue cycles, $N_{\text {sensor, }}$ is represented as the function of the normalized crack length, $\alpha$, and the strain amplitude, $\Delta \varepsilon_{\text {sensor, }}$ as:

$$
\begin{aligned}
& N_{\text {sensor }}= \\
& \frac{W}{C} \int_{\alpha_{0}}^{\alpha_{1}}\left[\frac{d \alpha}{\left\{\Delta \varepsilon_{\text {sensor }} E_{\text {sensor }}(1-R)^{\beta_{w}-1} \sqrt{\pi W \alpha} f(\alpha)\right\}^{m}-\Delta K_{t h 0}{ }^{m}}\right] .
\end{aligned}
$$


To confirm the feasibility of the proposed method, fatigue cycles were estimated using Eq. (17) and compered with experimental data for smooth (W10-H40) and side-grooved (W10-H40) under the assumption that the sensor is subjected to cyclic loading with strain ratio of $R=0.5$. The estimated value of fatigue cycles are shown in Figs. 8(a) and 8 (b), where the correlation coefficients for smooth (W10$\mathrm{H} 40$ ) and side-grooved (W10-H40) are 0.988 and 0.967 , respectively. Therefore, it was shown that fatigue cycles can be estimated using smart patch.

\subsection{Prediction of Stress Amplitude}

The detectable range of stress amplitude is considered under the same assumption as above. The minimum value of detectable stress amplitude in structure $\left(\Delta \sigma_{\text {strucuture, min }}\right)$ is given by substituting $\Delta K_{\mathrm{th}}=2.53 \mathrm{MPa} \cdot \mathrm{m}^{1 / 2}$ into Eqs. (2) and (15) as,

$$
\Delta \sigma_{\text {structure, min }}=\frac{E_{\text {structure }} \Delta K_{\mathrm{th}}}{E_{\text {sensor }} \sqrt{\pi W \alpha_{i}} \cdot g\left(\alpha_{i}, \beta\right)},
$$

where $\alpha_{i}$ is initial normalized crack length. In the same manner, the maximum value of detectable stress amplitude ( $\left.\Delta \sigma_{\text {strucuture, max }}\right)$ can be obtained by plugging the value of the critical stress intensity factor range $\left(\Delta K_{\mathrm{fc}}\right)$ in Eq. (2). The $\Delta K_{\mathrm{fc}}$ was arbitrarily defined as the value of $\Delta K$ at which the value of $d a / d N$ corresponds to $10^{-6} \mathrm{~m} /$ cycle, because it becomes difficult to measure the crack growth rate in current system. Figure 9(a) shows measurable stress range of smart patch as a function of free length when the width of sensor is fixed to $10 \mathrm{~mm}$, under the assumption that the steel structure $\left(E_{\text {strucuture }}=200 \mathrm{GPa}\right)$ is subjected to cyclic loading with strain ratio of $R=0.5$. It was found that the detectable stress range is easily influenced by sensor length. By contrast, when the length is fixed to $10 \mathrm{~mm}$, the width of the sensor has an insignificant effect on the detectable stress as shown in Fig. 9(b). In the region of these figures, the minimum detectable stress was estimated to be $11 \mathrm{MPa}$ when $L=80 \mathrm{~mm}$ and $W=10 \mathrm{~mm}$, and the maximum detectable stress was estimated to be $632 \mathrm{MPa}$ when $L=5 \mathrm{~mm}$ and $W=$ $10 \mathrm{~mm}$. This extension of the measurement range in the smart patch allows us to evaluate fatigue damage over a wide range of infrastructures. In fatigue assessment for steel highway bridge in Japan, ${ }^{29)}$ fatigue limits of steel components and welded joints are assumed between 30-270 MPa, and the corresponding fatigue strength at $10^{5}$ cycles are categorized between approximately 100-600 MPa. These stress conditions can be monitored by newly developed smart patch.

In actual situation of structural components, stress amplitude is not constant, and the stress ratio changes as time progresses. It should be noticed that the threshold stress intensity factor range of the sensor is affected by the strain ratio (Fig. 4). Therefore, it is necessary to evaluate the effect of stress ratio on the prediction of stress amplitude in the smart patch. The modified Walker's model was found to describe the R-ratio effect well for a number of materials in ambient air, ${ }^{30,31)}$
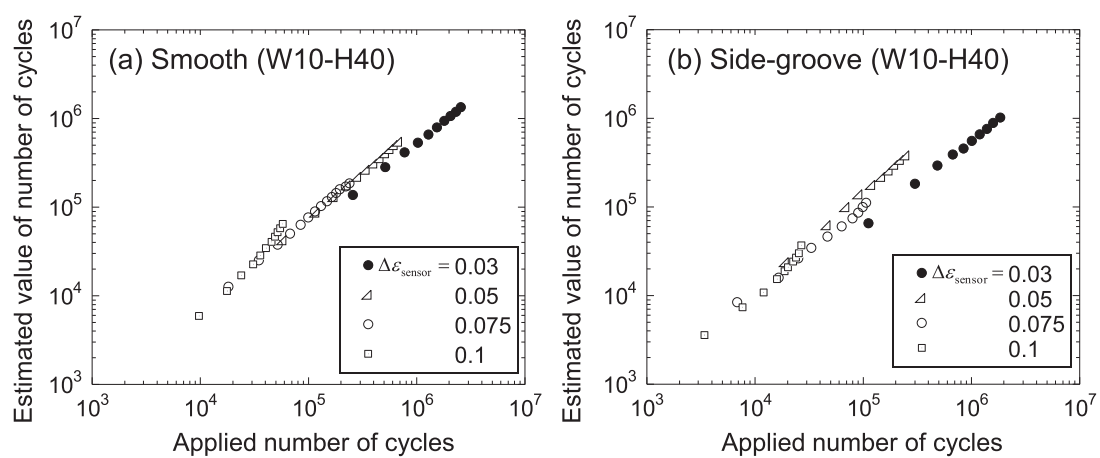

Fig. 8. Prediction of fatigue life based on Eq. (17). (a) Smooth specimen (W10-H40); (b) side-grooved specimen (W10-H40).
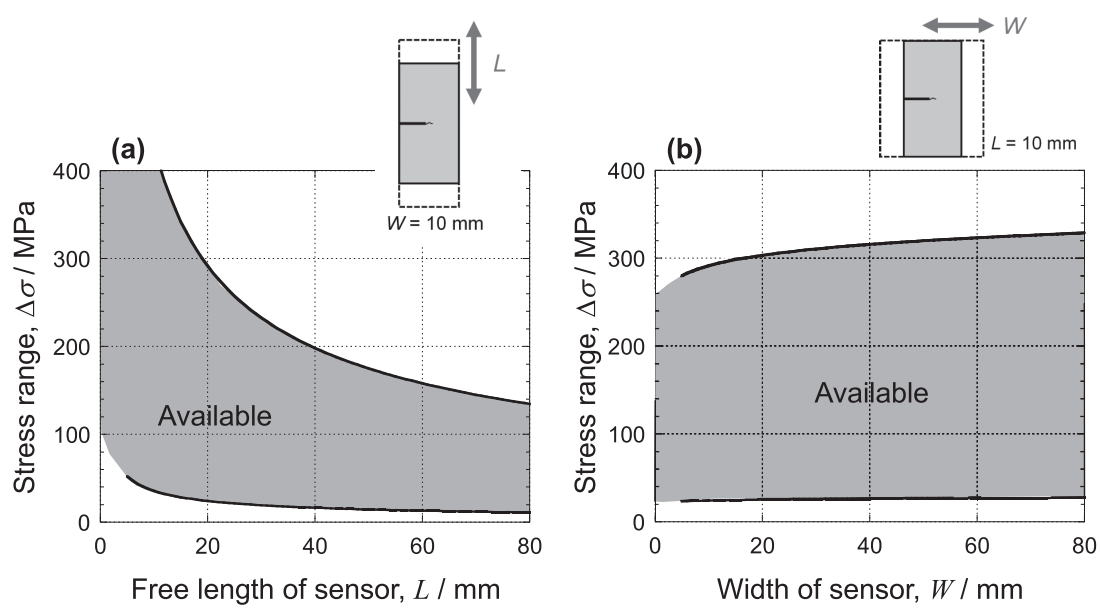

Fig. 9. Measurable stress range of smart patch in the case of $R=0.5$. Effect of (a) free length; (b) width of sensor. 


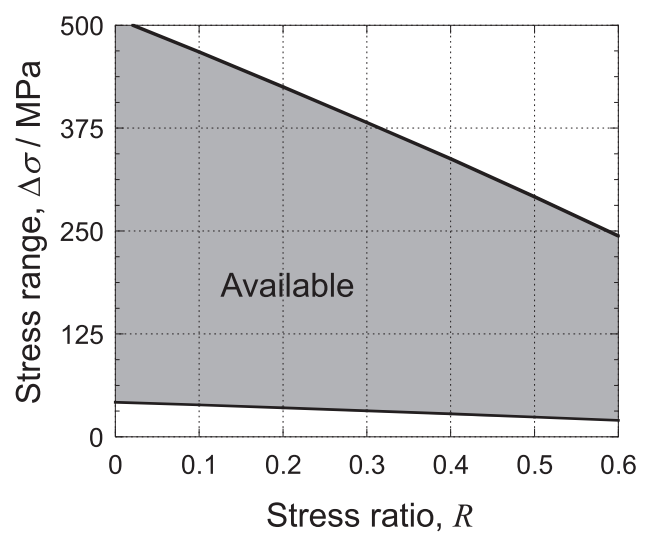

Fig. 10. Measurable stress range of smooth specimen (W5-H20) as a function of stress ratio.

$$
\Delta K_{\mathrm{th}}=\Delta K_{\mathrm{th} 0}[1-\langle R\rangle]^{1-\beta_{w}}
$$

The symbol $\langle x\rangle$ is the Macaulay bracket, which is equal to $x$ if $x>0$ and 0 if $x<0$. For the current sensor, two constants $K_{\text {th } 0}$ and $\beta_{w}$ in the above equation were identical to that of Eq. (11), which are determined experimentally for three Rratios (Fig. 6). The detectable range of stress amplitude for various stress ratios is calculated by following equations:

$$
\Delta \sigma_{\text {structure, } \max }=\frac{E_{\text {structure }}(1-R)^{1-\beta_{w}}}{E_{\text {sensor }} \sqrt{\pi W \alpha_{i}} \cdot g\left(\alpha_{i}, \beta\right)}\left(\frac{10^{-6}}{C}+\Delta K_{\text {th } 0}{ }^{m}\right)^{1 / m}
$$

$$
\Delta \sigma_{\text {structure, } \min }=\frac{E_{\text {structure }} \Delta K_{\mathrm{th} 0}[1-\langle R\rangle]^{1-\beta_{w}}}{E_{\text {sensor }} \sqrt{\pi W \alpha_{i}} \cdot g\left(\alpha_{i}, \beta\right)} .
$$

Figure 10 shows the description of the R-ratio effects on the detectable stress amplitude for smooth specimen (W5-H20). It indicates that the measurement range of the smart patch can be affected by stress ratio.

\section{Conclusions}

In the present study, fatigue crack growth behavior in thin pure copper sheet with pre-crack was evaluated under strain-controlled fatigue testing in order to obtain the sensor characteristics of smart stress-memory patch and the following conclusions were obtained.

(1) The fatigue crack growth rate of the sensor increased with increasing sensor length and presence of side-groove under the same test condition, because the shape factor of stress intensity factor increased with increasing aspect ratio $(\beta)$ and presence of side-groove.

(2) To characterize the initiation as well as the stable growth of fatigue cracks, relationship between stress intensity factor range and crack growth rate of the ED-Cu sensor was successfully fitted to one equation regardless of strain amplitude, strain ratio and specimen shape.

(3) The influence of side-groove on fatigue crack retardation was not observed in the sensor. It indicates that the crack growth rate of thin specimen that has the other shape parameters such as length, width and groove could be predicted based on shape factor calculated by FEM and material constants.
(4) Using Kohout equation, the fatigue cycles was represented as the function of the normalized crack length in the sensor and the strain amplitude. It was shown that cyclic number of structure can be estimated from the crack length of ED-Cu specimen under the simple assumption that the sensor is perfectly bonded on the structure.

(5) The detectable range of stress amplitude was improved by changing the sensor shape. The longer sensor detects the smaller stress amplitude, and the shorter sensor provides a wider range of stress measurement. The width of the sensor has an insignificant effect on the detectable stress.

(6) The measurement range of the smart patch can be affected by stress ratio. The dependence of stress ratio on detectable stress was expressed by modified Walker's model.

\section{Acknowledgement}

This study was financially supported by the Iron and Steel Institute of Japan (ISIJ) and Japan Society for the Promotion of Science KAKENHI (Grant Number 25889014).

\section{REFERENCES}

1) D. E. Adams: Health Monitoring of Structural Materials and Components, Wiley, New Jersey, (2007), 1.

2) J. P. Lynch and K. J. Loh: A Summary Review of Wireless Sensors and Sensor Networks for Structural Health Monitoring, Shock and Vibration Digest, Sage Publications, California, (2006), 1.

3) N. Xu, S. Rangalwala, K. Chintalapudi, D. Ganeshan, A. Broad, R. Govindan and D. Estrin: Proc. of 2nd Int. Conf. on Embedded Networked Sensor Systems, Association for Computing Machinery, New York, (2004), 13.

4) S. N. Pakzad, S. Kim, G. L. Fenves, S. D. Glaser, D. E. Culler and J. W. Demmel: Proc. of 5th Int. Workshop on Structural Health Monitoring, Stanford University, California, (2005), 125.

5) S. Kim, S. Pakzad, D. Culler, J. Demmel, G. Fenves, S. Glaser and M. Turon: Proc. of 6th Int. Conf. on Information Processing in Sensor Networks, ACM Press, New York, NY, (2007), 254.

6) S. Nambu and M. Enoki: ISIJ Int., 47 (2007), 1687.

7) S. Nambu and M. Enoki: ISIJ Int., 51 (2011), 88.

8) T. Shiraiwa and M. Enoki: ISIJ Int., 51 (2011), 250.

9) T. Shiraiwa and M. Enoki: ISIJ Int., 51 (2011), 1480.

10) S. Nambu and M. Enoki: Mater. Trans., 48 (2007), 1244.

11) Y. Fujino, S. Nambu and M. Enoki: Mod. Phys. Lett. B, 22 (2008), 1105.

12) H. D. Merchant, M. G. Minor and Y. L. Liu: J. Electron. Mater., 28 (1999), 998

13) M. Judelewicz, H. U. Künzi, N. Merk and B. Ilschner: Mater. Sci. Eng. A, 186 (1994), 135.

14) M. Gonzalez, F. Axisa, M. V. Bulcke, D. Brosteaux, B. Vandevelde and J. Vanfleteren: Microelectron. Reliab., 48 (2008), 825.

15) T. Hanlon, E. D. Tabachnikova and S. Suresh: Int. J. Fatigue, 27 (2005), 1147.

16) ASTM E647 00, Standard Test Method for Measurement of Fatigue Crack Growth Rates, ASTM, WI, (2005), 1.

17) Y. Murakami: Stress Intensity Factor Handbook, Pergamon Press, Oxford, (1987), 99.

18) P. J. Torvik: J. Appl. Mech., 46 (1979), 611.

19) P. C. Paris and F. Erdogan: Trans. ASTM J. Basic. Eng., 85 (1963), 528.

20) P. C. Paris, M. P. Gomez and W. E. Anderson: Trend Eng., 13 (1961),

21) M. Klesnil and P. Lukas: Engng. Fract. Mech., 4 (1972), 77.

22) J. Kohout: Int. J. Fatigue, 21 (1972), 813.

23) K. Ortiz and A. S. Kiremidjian: Eng. Fract. Mech., 24 (1986), 657.

24) K. Ortiz and A. S. Kiremidjian: Eng. Fract. Mech., 29 (1988), 317.

25) J.-K. Kim and D.-S. Shim: Int. J. Fatigue, 22 (2000), 611.

26) N. Minegishi and Y. Hagiwara: Bull. JSME, 29 (1986), 334

27) J. P. Hess, A. F. Grandt and A. Dumanis: Fatigue. Fract. Eng. M., 6 (1983), 189.

28) G. Green and J. F. Knott: Met. Technol., 2 (1975), 422

29) Japanese Society of Steel Construction (JSSC): JSSC Tech. Rep., 32 (1995), 1.

30) D. Kujawski: Int. J. Fatigue, 23 (2001), Supplement 1, 239.

31) L. Weng, J. Zhang, S. Kalnaus, M. Feng and Y. Jiang: Int. J. Fatigue, 48 (2013), 156. 